\title{
Ecología de Phyllodactylus angustidigitus y P. gerrhopygus (Squamata: Phyllodactylidae) de la Reserva Nacional de Paracas, Perú
}

\author{
Ecology of Phyllodactylus angustidigitus and P. gerrhopygus (Squamata: \\ Phyllodactylidae) from the Reserva Nacional de Paracas, Peru
}

1 Departamento de Herpetología, Museo de Historia Natural. Universidad Nacional de Mayor de San sidad Nacional de Mán. Lima, Perú.

2 Laboratorio de Estudios en Biodiversidad (LEB). Departamento chíla de Biologia y Fisiologia. Facultad de Ciencias y Filosofia. Universidad Peruana Cayetano Heredia (UPCH). Lima, Perú.

3 Departamento de Ecologia. Instituto de Biologia. Universidade do Estado do Rio de Janeiro (UERJ). Rio de Janeiro, Brasil.

Email José Pérez Z. jose.perez.z@upch.pe $\begin{array}{ll}\text { Presentado: } & 12 / 02 / 2010 \\ \text { Aceptado: } & 23 / 07 / 2010\end{array}$
José Pérez Z.1,2,3 y Katya Balta ${ }^{2}$

\section{Resumen}

Actualmente se desconocen muchos aspectos de la ecología de Phyllodactylus angustidigitus (Dixon \& Huey 1970) y $P$. gerrhopygus (Weigmann, 1834). En el presente trabajo investigamos algunos aspectos del nicho de $P$. angustidigitus y $P$. gerrhopygus en la Reserva Nacional de Paracas (RNP). Se evaluó y comparó la distribución, uso de hábitats (nicho espacial), horarios de actividad (nicho temporal) y dieta (nicho trófico) de estas dos especies de gecónidos. Adicionalmente, se estimó la oferta de recursos alimentarios mediante el uso de trampas pit-fall. Phyllodactylus angustidigitus estuvo presente en la mayoría de las localidades evaluadas, y fue registrado en los hábitats de desierto y lomas. Phyllodactylus gerrhopygus fue registrado únicamente en la Península de Paracas y en el oasis de Sta. Cruz, en los hábitats de desierto, lomas y oasis. Ambas especies fueron registradas en actividad sólo en horarios nocturnos. La dieta de $P$. angustidigitus y $P$. gerrhopygus estuvo caracterizada principalmente por el consumo de insectos. Ambas especies presentaron dietas de tipo generalistas, debido a la amplitud del nicho trófico, y oportunistas, debido a la semejanza en la composición de sus dietas con la oferta estimada de recursos alimentarios en el medio ambiente. Las dietas de ambas especies fueron semejantes. Adicionalmente, la cercanía filogenética entre $P$. angustidigitus y $P$. gerrhopygus producen semejanzas en el nicho espacial (uso de hábitats), temporal (horarios de actividad), y en sus dietas. Estas importantes similitudes en el uso de los recursos del medio, pueden derivar en una potencial competencia por los recursos de su medio ambiente entre estos gecónidos simpátricos. La información proporcionada en este trabajo demuestra la necesidad de diseñar una adecuada estrategia para garantizar el estado de conservación de $P$. angustidigitus.

Palabras clave: Gecónidos; Paracas; uso de hábitats; actividad; dieta.

\section{Abstract}

Until now, ecology of Phyllodactylus angustidigitus and $P$. gerrhopygus are unknown. In this work, we researched some aspects of $P$. angustidigitus and $P$. gerrhopygus niches at National Reserve of Paracas (RNP). We evaluated and compared: distribution, habitat use (spatial niche), activity patterns (temporal niche) and diet (trophic niche), all these aspects in both geckonids species. We estimated resource supply through pitfall traps. Paracas geckonid, P. angustidigitus, has been present in most of the RNP evaluated locations, and it was reported in desert and hills habitats. Southern geckonid, P. gerrhopygus, was reported only in Paracas peninsula and Sta. Cruz, in desert, hills and oasis habitats. Both species were founded in activity at night time. $P$. angustidigitus and P. gerrhopygus diet consisted in insects. The diet of these species shows a generalist behavior, caused by the trophic niche amplitude, and an opportunistic behavior, explained with similarity between diet composition and environment resource supply. Diet of both species was similar. Additionally the phylogenetic proximity between $P$. angustidigitus y $P$. gerrhopygus produces spatial niche similarities (habitat use) and temporal niche similarities (activity patterns) and trophic niche (diet). These important similarities in resources use can be derived in a potential competition of environmental resources between these simpatric geckonids. The information provided in this paper shows the need to design an appropriate strategy to ensure the conservation status of $P$. angustidigitus.

Keywords: geckos; Paracas; use of habitat; activity; diet.

\section{Introducción}

En el Desierto Costero peruano se han registrado un total de nueve especies de gecónidos (Dixon \& Huey 1970, Carrillo \& Icochea 1995), todas pertenecientes al género Phyllodactylus (Familia Phyllodactylidae), las cuales presentan actividad nocturna (Dixon \& Huey 1970, Pérez \& Balta 2007). Se tiene poca información sobre la ecología de estas especies de Phyllodactylus; principalmente se han desarrollado investigaciones en aspectos de su ecología térmica (Werner et al. 1996, Pérez 2005), morfología (Carrillo et al. 1990) y sus dietas (Huey 1979, Pérez 2005, Jordán 2006, Catenazzi \& Donnelly 2007b). Huey (1979) analizó la comunidad de gecónidos del departamento de Piura, Jordán (2006) reportó los principales hábitos alimenticios de $P$. reissii para la Zona Reservada de Tumbes. Pérez (2005) reportó la dieta de P. lepidopygus en el ecosistema de Lomas en la Reserva Nacional de Lachay del departamento de Lima. Catenazzi y
Donelly (2007a) reportaron que la dieta de tres especies de gecónidos del desierto costero norte incluye nutrientes de origen marino y terrestre en sus dietas. Estos estudios destacan la dieta generalista de las especies de Phyllodactylus en el Perú, la cual está basada principalmente en insectos.

Phyllodactylus angustidigitus y Phyllodactylus gerrhopygus están presentes en la Reserva Nacional de Paracas (RNP) (Pérez \& Balta 2007), pero no existe información sobre su ecología. Pérez y Balta (2007) reportan información sobre el nicho trófico, espacial y temporal de los saurios diurnos de la RNP, sin embargo, no incluyen información de los gecónidos.

El gecónido de Paracas Phyllodactylus angustidigitus presenta una distribución restringida a la RNP y localidades próximas (Dixon \& Huey 1970), todas ubicadas dentro del Desierto Costero peruano. Por otro lado, el gecónido del sur $P$. gerrhopygus presenta una distribución amplia desde el norte de Chile 


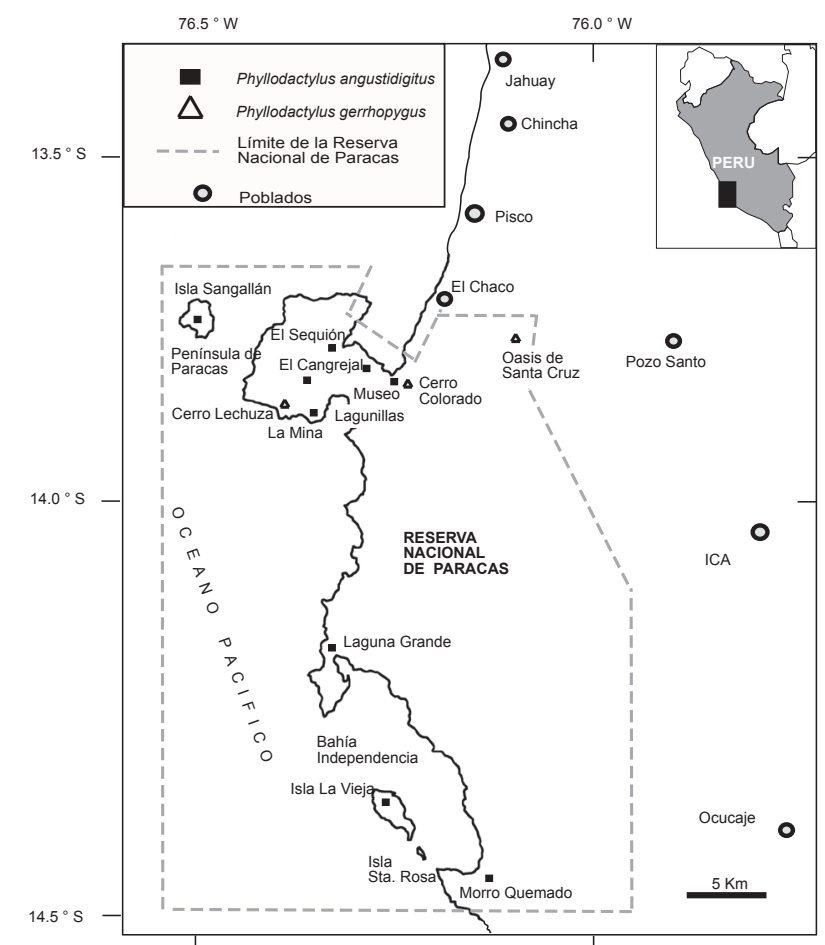

Figura 1. Área de estudio indicando las localidades con registros para Phyllodactylus angustidigitus y Phyllodactylus gerrhopygus en la Reserva Nacional de Paracas, Ica, Perú. Se indican algunos poblados próximos como referencias geográficas.

(Donoso-Barros 1966, Núñez \& Jaksic 1992) hasta el sur de Lima (Aguilar et. al 2007), que incluye registros dentro del Desierto Costero peruano, y localidades hasta los $2750 \mathrm{~m}$ de altitud en la ecoregión de Serranía Esteparia en el departamento de Arequipa (Zeballos et al. 2002). Por otro lado, cabe destacar que $P$. angustidigitus y $P$. gerrhopygus son reconocidas como especies filogenéticamente próximas (Dixon \& Huey 1970).

El presente trabajo tiene como objetivo proporcionar información y comparar algunos aspectos de los nichos espacial (uso de hábitats), temporal (horarios de actividad) y tróficos (dieta) de $P$. angustidigitus y $P$. gerrhopygus en la RNP.

\section{Material y métodos}

La evaluación fue realizada en la RNP (76¹6'15"W, $13^{\circ} 51^{\prime} 46^{\prime}$ 'S), ubicada en el Departamento de Ica, en la ecoregión del Desierto Costero Peruano (Fig. 1). La temperatura promedio anual en la RNP es de $18,7^{\circ} \mathrm{C}$, variando desde $15,5^{\circ} \mathrm{C}$ en agosto a $22^{\circ} \mathrm{C}$ en febrero, con una humedad relativa promedio anual de $82 \%$, y una precipitación prácticamente nula (INRENA 2002).

La evaluación se realizó en los meses de febrero, mayo, octubre y noviembre del 2000, en las localidades de La Mina, Lagunillas, Cerro Lechuza, El Cangrejal, Sequión, Cerro Colorado, Península, Laguna Grande, Morro Quemado, Oasis de Santa Cruz y las islas La Vieja y Sangayán. Todas estas localidades están ubicadas en la RNP. Se identificaron los hábitat de Playa, Desierto, Lomas, Oasis, siguiendo la clasificación propuesta por Pérez y Balta (2007), que incluyen detalles de estos hábitat. El hábitat de Playas fue evaluado en las localidades de El Cangrejal, Sequión, Lagunillas, La Mina, Laguna Grande, Morro Quemado, y en las islas La Vieja y Sangayán. El hábitat de Desierto fue evaluado en Cerro Colorado, El Cangrejal, Sequión, Lagunillas, La Mina,
Península (áreas en el centro de la Penínula de Paracas), Laguna Grande, Morro Quemado, alrededores de Santa Cruz, y en las islas La Vieja y Sangayán. El hábitat de Lomas fue evaluado en Cerro Lechuza, Morro Quemado, y en la isla Sangayán. El hábitat de Oasis fue evaluado en la localidad de Santa Cruz y en el área comprendida entre este punto y la playa de Santo Domingo.

Las localidades fueron evaluadas entre las 18:00 - 00:00 h Se realizó un esfuerzo de muestreo semejante en cada uno de los hábitat identificados. Los datos recopilados incluyen información de registros visuales y capturas. Para cada saurio observado registramos la especie, hábitat y hora del registro. A partir de esta información analizamos el nicho espacial y temporal de Phyllodactylus angustidigitus y Phyllodactylus gerrhopygus.

Utilizamos el índice de Simpson (Krebs 1999) para estimar la amplitud de los nichos temporal y espacial (uso de hábitat y actividad) de cada especie de gecónido, mediante la fórmula: $\mathrm{B}_{i j}$ $=1 / \sum \mathrm{p}_{i}^{2}$, donde $\mathrm{p}_{i}$ es la proporción del recurso i. Adicionalmente estimamos la similaridad en el nicho espacial y temporal entre estos dos gecónidos mediante el índice de superposición (Pianka 1986):

$$
O_{\mathrm{jk}}=\frac{\sum_{i=1}^{n} \text { pij pik }}{\sqrt{\sum_{i=1}^{n} p i j^{2} \sum_{i=1}^{n} p i k^{2}}}
$$

Donde $i$ es el tipo de recurso, y $j$ y $k$ son las especies de saurios. Los valores de esta fórmula varían entre 0 (ausencia de superposición) y 1 (completa superposición).

Los individuos utilizados para el análisis de los nichos tróficos fueron capturados únicamente entre las 19:00 - 22:00 h para reducir la probabilidad de registrar individuos con estómagos vacíos o contenido estomacal muy digerido. Todos los individuos fueron colectados manualmente, sacrificados en alcohol al 70\%, fijados en formol 10\% y posteriormente disectados en el laboratorio. El material colectado fue depositado en el Departamento de Herpetología del Museo de Historia Natural de la Universidad Nacional Mayor de San Marcos.

Se analizó el contenido estomacal con ayuda de un microscopio estereoscópico. Los ítems consumidos fueron identificados hasta el nivel de Orden. Todas las partes de artrópodos no identificadas fueron agrupadas en la categoría denominada PANI (Partes de Artrópodos No Identificados). Todas las partes de plantas fueron incluidas en la categoría Material Vegetal. Se estimó el número, frecuencia y volumen de cada ítem alimentario. El volumen de cada ítem alimentario fue estimado mediante la fórmula del ovoide-esfeoride (Dunham 1983): 4/3 $\pi$ (largo del ítem/2) * (ancho del item/2) ${ }^{2}$. Las dimensiones de cada ítem fueron medidas con un calibrador Vernier $(0,1 \mathrm{~mm}$ de precisión). Adicionalmente, se estimó la importancia de cada ítem alimentario para cada especie de gecónido mediante el Índice de importancia (Powell et al. 1990) mediante la fórmula: $\mathrm{I}_{x}=$ (\% Número + \% Volumen + \% Frecuencia $) / 3$.

Se estimó la amplitud del nicho trófico mediante el índice de Simpson. También se comparó el número de tipos de ítems alimentarios, el número y largo de presas, así como la amplitud 
promedio del nicho trófico entre $P$. angustidigitus y $P$. gerrhopygus mediante la prueba de Mann-Whitney (Zar 1999). No se utilizaron pruebas paramétricas ya que los datos no satisficieron las condiciones para ese tipo de análisis. Adicionalmente, se comparó la composición de la dieta de estas dos especies mediante la prueba de Kolmogorov-Smirnov (Zar 1999).

La similaridad entre las dietas de estos gecónidos fue estimada mediante el índice de superposición de Pianka, para los valores del número de presas, así como para los estimados de volúmenes. La superposición estimada puede evidenciarnos un patrón en la comunidad evaluada, por ejemplo, estas especies podrían presentar una baja superposición en el nicho trófico debido a una activa división de los recursos. Sin embargo, un reducido valor de superposición también puede ser un resultado del azar. El programa Ecosim 7.0 (Gotelli \& Entsminger 2010), basado en modelos nulos de simulación, nos permite diferenciar entre patrones reales y resultados producto del azar. Por medio del Ecosim se estimó si el valor de superposición calculado entre $P$. angustidigitus y $P$ gerrhopygus es significativo, es decir, que reflejaría una patrón real en la relación entre estas dos especies de gecónidos, o sería producto del azar. El análisis por modelos nulos proporciona cuatro tipos de algoritmos para generar sus estimados. Para nuestro análisis se eligió el algoritmo RA3, siguiendo la recomendación de Winemiller y Pianka (1990). El algoritmo RA3 mantiene constante la amplitud de nicho y varia la posición de los ceros dentro de cada columna de la matriz de datos (tipo de presas), y debido a esto presenta adecuadas características estadísticas para detectar patrones no aleatorios de superposición de nicho (Winemiller y Pianka 1990). Para este análisis, la matriz de datos estuvo basada en la proporción de consumo de cada tipo de ítem alimentario para $P$. angustidigitus y $P$. gerrhopygus. Se eligió trabajar con las proporciones y no simplemente con el número de individuos registrado, para poder realizar mejor las comparaciones debido a la diferencia de tamaños muestreales entre las dos especies de gecónidos.

Adicionalmente, realizamos una colecta de invertebrados para conocer la oferta de presas que ofrece el medio ambiente a estos saurios. Se instalaron 1 - 2 trampas pit-fall en los lugares de captura de cada uno de los gecónidos. Estas trampas fueron vasos de plástico de $250 \mathrm{~mL}$ enterrados al ras en el sustrato, con agua y detergente, para romper la tensión superficial y capturar presas pequeñas. Estas trampas fueron revisadas dos veces por día, y estuvieron activas dos días como tiempo mínimo. Comparamos la composición de las dietas de Phyllodactylus angustidigitus y $P$. gerrhopygus con las presas capturadas en el ambiente (oferta del medio) mediante la prueba de Kolmogorov-Smirnov (Zar 1999). Para esta comparación no son consideradas en el análisis las categorías PANI y Material Vegetal de los contenidos estomacales de ambas especies de gecónidos, ya que su oferta en el medio no pudieron ser estimadas por los métodos empleados. Este análisis nos proporcionará información si las dietas de estas dos especies de gecónidos son principalmente oportunistas o selectivas. La composición de la dieta de un gecónido puede ser semejante a la oferta de invertebrados del medio (presas), y la dieta de este reptil puede ser considerado oportunista, ya que consumiría en mayor proporción lo más abundante en su ambiente. En caso contrario, si la composición de la dieta del gecónido es significativamente diferente a la oferta de recursos alimentarios de su medio, este reptil estaría seleccionando activamente las presas que consume.

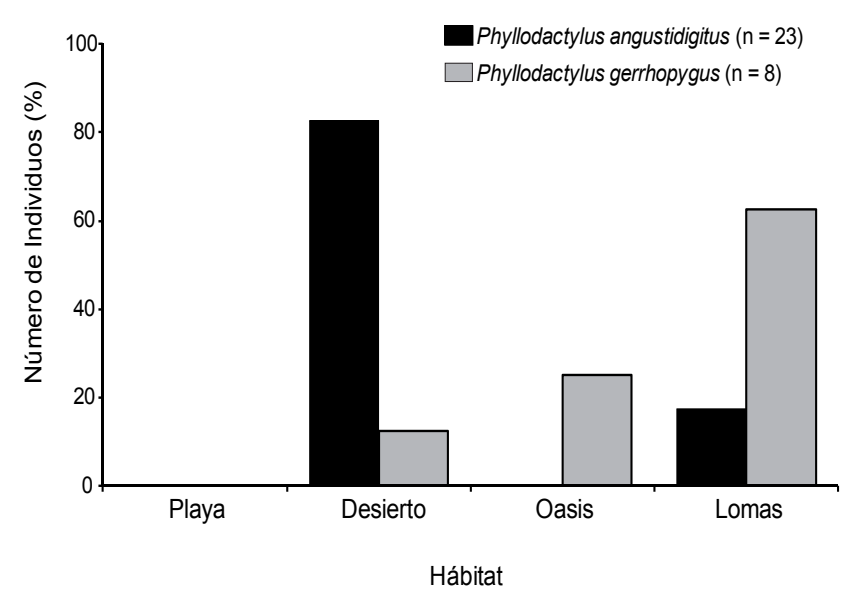

Figura 2. Porcentaje del número de individuos de Phyllodactylus angustidigitus y Phyllodactylus gerrhopygus registrados en cada tipo de hábitat en la Reserva Nacional de Paracas, Ica, Perú.

\section{Resultados}

Se registraron un total de 23 Phyllodactylus angustidigitus en las localidades de La Mina, Lagunillas, El Cangrejal, Sequión, Cerro Colorado, Península, Laguna Grande, Morro Quemado, y las islas La Vieja y Sangayán (Fig. 1). Se registraron un total de ocho Phyllodactylus gerrhopygus en las localidades de Cerro Colorado, Cerro Lechuza, Oasis de Santa Cruz (Fig. 1). La única localidad donde se registraron a ambas especies fue Cerro Colorado.

Phyllodactylus angustidigitus fue registrado en dos de los cuatro hábitats identificados (Desierto y Lomas), mientras que P. gerrhopygus fue registrado en tres de estos hábitats (Desierto, Lomas y Oasis) (Fig. 2). No se registraron individuos de ninguna especie en el hábitat de Playa. La amplitud del nicho espacial fue $\mathrm{B}_{i j}=1,4$ y $\mathrm{B}_{i j}=2,1$ para $P$. angustidigitus y $P$. gerrhopygus respectivamente. Se registró un valor bajo de superposición en el nicho espacial $\left(\mathrm{O}_{j k}=0,4\right)$ entre estos dos gecónidos.

Se obtuvieron un número reducido de registros de $P$. gerrhopygus para el análisis del nicho temporal $(\mathrm{n}=5)$, sin embargo, se presentan los resultados para fines comparativos, y se discutirán considerando sus posibles limitaciones. Ambas especies presentarían horarios de actividad semejante, limitándose a horarios nocturnos, principalmente a inicios de la noche (Fig.

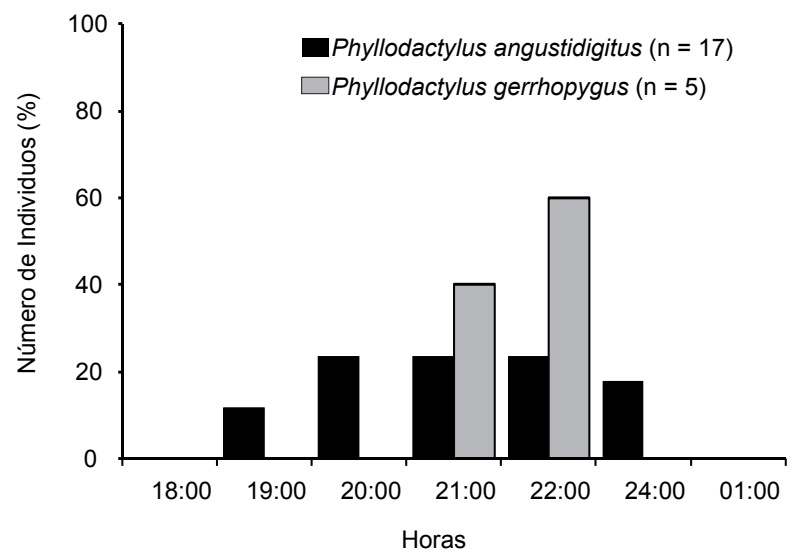

Figura 3. Porcentaje del número de individuos de Phyllodactylus angustidigitus y Phyllodactylus gerrhopygus registrados en cada intervalo horario en la Reserva Nacional de Paracas, Ica, Perú. 


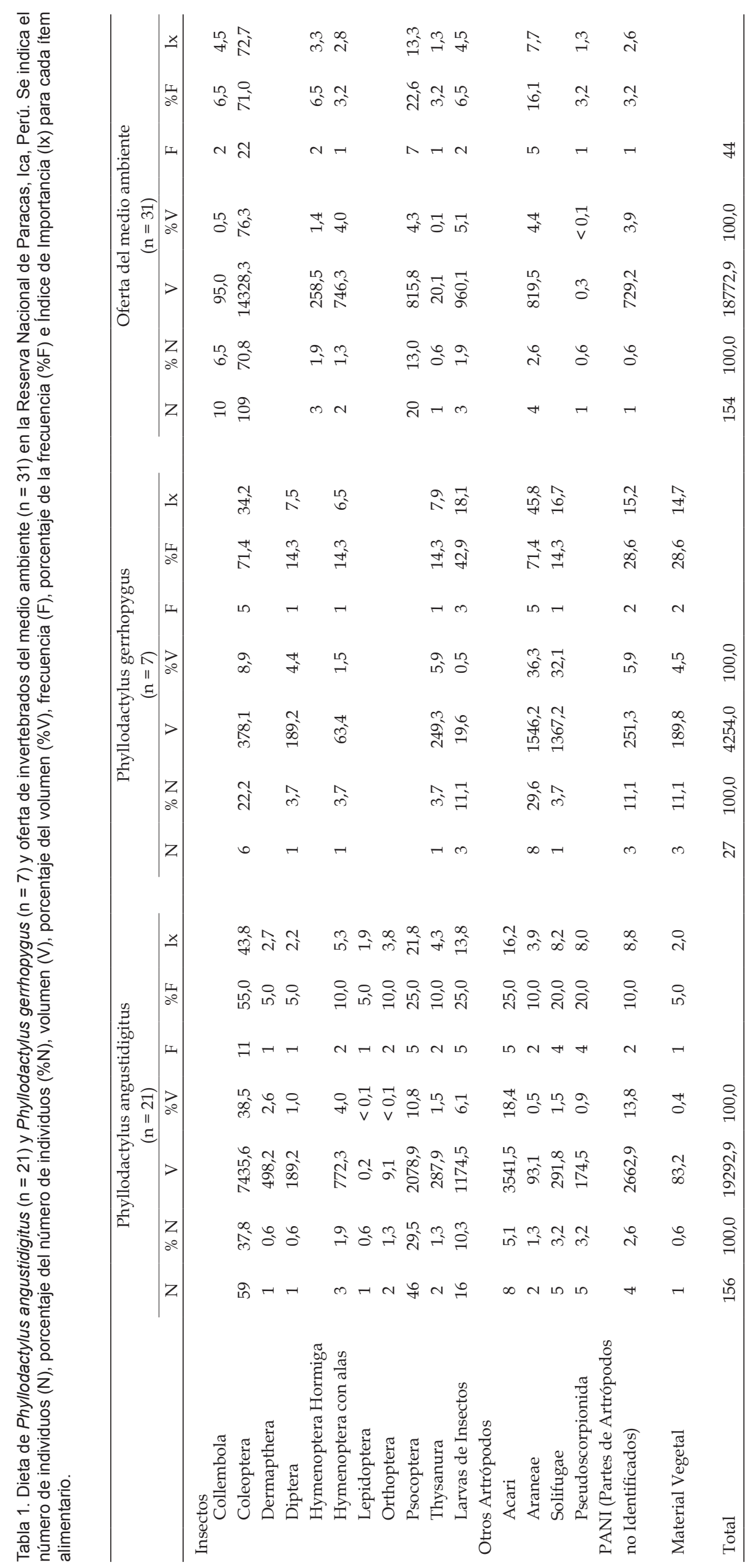


3). La amplitud del nicho temporal fue $\mathrm{B}_{i j}=4,7$ y $\mathrm{B}_{i j}=1,9$ para $P$. angustidigitus y $P$. gerrhopygus respectivamente. Se registró un valor bajo de superposición en el nicho espacial $\left(\mathrm{O}_{j k}=0,3\right)$. Este valor está fuertemente influenciado por el reducido número de registros de $P$. gerrhopygus.

Para la evaluación del nicho trófico se analizaron un total de 21 contenidos estomacales de $P$. angustidigitus y siete de $P$. gerrhopygus. Se registró un único individuo de $P$. angustidigitus con el estómago vacío. En seis estómagos de $P$. angustidigitus se encontraron piedras pequeñas. No se registraron piedras en los estómagos analizados de $P$. gerrhopygus.

El principal ítem alimentario en términos numéricos para P. angustidigitus fueron los coleópteros (37,8\%), seguido de los psocópteros $(29,5 \%)$ y larvas de insectos $(10,3 \%)$ (Tabla 1$)$. En térmicos volumétricos los principales ítems alimentarios fueron los coleópteros (38,5\%), seguido de los ácaros (18,4\%) y psocópteros (10,8\%) (Tabla 1). Los ítems más frecuentes fueron los coleópteros (55,0\%), seguidos de los psocópteros, larvas de insectos y ácaros (todos con 25,0\%) (Tabla 1). Según el índice de importancia (I) los ítems alimentarios más importantes para $P$. angustidigitus fueron los coleópteros, seguido de los psocópteros, ácaros y larvas de insectos (Tabla 1). El tamaño promedio de los ítems consumidos por $P$. angustidigitus fue de 40,0 $\pm 21,3 \mathrm{~mm}(\mathrm{n}=173)$. La amplitud del nicho trófico de $P$. angustidigitus fue de $\mathrm{B}_{i j}=4,1$.

En el caso de $P$. gerrhopygus, los principales ítems alimentarios en términos numéricos fueron las arańas $(29,6 \%)$, seguido de los coleópteros $(22,2 \%)$, larvas de insectos y material vegetal (ambos 11,1\%) (Tabla 1). En térmicos volumétricos los principales ítems alimentarios fueron las arañas (36,3\%), seguido de los solífugos $(32,1 \%)$ y coleópteros $(8,9 \%)$ (Tabla 1$)$. Los ítems más frecuentes fueron las arańas y coleópteros (ambos 71,4\%), y larvas de insectos $(42,9 \%)$ (Tabla 1$)$. Según el índice de importancia $\left(\mathrm{I}_{x}\right)$ los ítems alimentarios más importantes para $P$. gerrhopygus fueron las arañas, seguido de los coleópteros, larvas de insectos y solífugos (Tabla 1). El tamaño promedio de los ítems consumidos por $P$. gerrhopygus fue de 45,0 $\pm 30,4 \mathrm{~mm}(\mathrm{n}=27)$. La amplitud del nicho trófico de P. gerrhopygus fue de $\mathrm{B}_{i j}=5,6$.

No se registraron diferencias significativas entre el número de tipos de ítems consumidos por $P$. angustidigitus $(2,24)$ y $P$. gerrhopygus $(2,72)$ (Prueba Mann-Whitney $\mathrm{U}=59,5 ; \mathrm{p}=0,44)$, número de presas consumidas por $P$. angustidigitus $(14,0)$ y $P$. gerrhopygus $(3,6)$ (Prueba Mann-Whitney $U=103,0 ; \mathrm{p}=0,12$ ), ni en el tamaño de ítems consumidos entre estas dos gecónidos (Prueba Mann-Whitney U = 2185,50; p = 0,59). Sin embargo, la amplitud promedio del nicho trófico de $P$. angustidigitus $\left(\mathrm{B}_{i j}=\right.$ $1,75)$ fue significativamente menor que el de $P$. gerrhopygus $\left(\mathrm{B}_{i j}\right.$ $=3,28$ ) (Prueba Mann-Whitney $U=33,50 ; p=0,02$ ).

La superposición en términos numéricos entre las dietas de $P$. angustidigitus y $P$. gerrhopygus registró un valor de $\mathrm{O}_{j k}=0,4$. A partir de las simulaciones realizadas se observa que las dietas en términos numéricos de estos gecónidos no son significativamente diferentes de los valores de superposición producidos al $\operatorname{azar}(\mathrm{p}=0,16)$.

La superposición en términos volumétricos entre las dietas de $P$. angustidigitus y $P$. gerrhopygus registró un valor de $\mathrm{O}_{j k}=0,2$. De manera semejante a lo observado en el análisis numérico, las dietas en términos volumétricos de estos gecónidos no son significativamente diferentes de los valores producidos al azar $(\mathrm{p}=0,53)$. Por lo tanto, la superposición en sus dietas presentan pequeños valores, sin embargo, estos no serían producto de la interacción entre estas dos especies de gecónidos para dividir el uso de los recursos alimentarios, si no serían producto del azar.

En el análisis en términos numéricos y volumétricos de las dietas de $P$. angustidigitus y $P$. gerrhopygus no se registraron diferencias significativas en las proporciones que consumieron los tipos de presas $(\mathrm{D}=0,400 ; \mathrm{p}=0,1346$ para ambos casos).

En la oferta de invertebrados del medio ambiente se observó una predominancia de los coleópteros en número, volumen y frecuencia (Tabla 1), siendo la presa principal $\left(\mathrm{I}_{x}=72,3 \%\right)$, seguido por los psocópteros y arańas (Tabla 1). Cabe mencionar que en las trampas se registraron colémbolos y hormigas, invertebrados no registrados para ninguna de las dietas de las dos especies de gecónidos. No se registraron solífugos en las trampas evaluadas, sin embargo, este tipo de artrópodo fue una presa considerablemente frecuente para $P$. angustidigitus y $P$. gerrhopygus (Tabla 1).

En el análisis en términos numéricos, las dietas de $P$. angustidigitus y $P$. gerrhopygus no fueron significativamente diferentes a la oferta de invertebrados registrada en el medio ambiente $(\mathrm{D}=0,267 ; \mathrm{p}=0,589$ para ambos casos). De manera similar, en términos volumétricos las dietas de $P$. angustidigitus y $P$. gerrhopygus no fueron significativamente diferentes a la oferta de invertebrados registrada en el medio ambiente $(D=0,313$; $\mathrm{p}=0,348 ; \mathrm{D}=0,197 ; \mathrm{p}=0,3944$ respectivamente). Estos resultados muestran que ambas especies de gecónidos consumirían en mayor proporción las presas más abundantes en su medio ambiente, por lo que presentan una dieta de tipo oportunista.

\section{Discusión}

A partir de los registros conocidos a la fecha para el Perú, Phyllodactylus gerrhopygus presenta una distribución continua y paralela al litoral desde el departamento de Tacna (sur del Perú) hasta San Juan de Marcona, en el sur del departamento de Ica, y a partir de este punto se separa ligeramente del litoral hasta la playa Jahuay (departamento de Lima), al norte de la RNP (Dixon \& Huey 1970, Fig. 2, pag. 15). A partir de Jahuay la distribución hacia el norte nuevamente se aproxima al litoral (Dixon y Huey, 1970), llegando al sur del departamento de Lima (Aguilar et al., 2007). El tramo de litoral sin registros de $P$. gerrhopygus corresponde a la RNP. A partir de la presente evaluación observamos que la distribución de P. gerrhopygus en la RNP parece seguir una franja desde Cerro Lechuza, al oeste de la Península de Paracas, hasta el Oasis de Sta. Cruz en el límite este de esta reserva. Esta especie ha sido registrada anteriormente en Pozo Santo (Dixon y Huey, 1970) al este de la RNP (Fig. 1), localidad que daría continuidad a la mencionada distribución en franja, y se conectaría con las poblaciones próximas al valle de Ica. Dixon y Huey (1970) no registraron a P. gerrhopygus en la RNP, sin embargo, a partir de los registros en la presente evaluación se puede sugerir que esta especie estaría ampliando su distribución hacia zonas dentro de esta reserva. Por otro lado, la distribución de Phyllodactylus angustidigitus está limitada a la RNP, y ha sido registrado desde la Península de Paracas hasta Morro Quemado (Fig. 1).

Cabe mencionar, que Dixon y Huey (1970) proponen la hipótesis que $P$. angustidigitus tendría una distribución limitada 
debido a la reciente aparición como especie, y por lo tanto, estaría iniciando la ampliación de su distribución a partir de la Península de Paracas hacia otras zonas en la RNP. Phyllodactylus gerrhopygus presenta una distribución latitudinal mucho mayor, llegando hasta el norte de Chile (Núñez y Jacsik 1992), y alcanzando los $2750 \mathrm{~m}$ de altitud en Arequipa (Zeballos et al. 2002) y 3000 m de altitud. en Tacna (Pérez datos no publicados). Las considerables diferencias en el tamaño de sus distribuciones, sugieren un mayor grado de adaptación de $P$ gerrhopygus a variaciones en el ambiente.

Adicionalmente, cabe destacar que $P$. gerrhopygus ha sido reportado a lo largo de su distribución en algunas zonas con importante presencia de vegetación, como las Lomas de Matarani y Atiquipa en Arequipa (Zeballos et al. 2002), y Morro Sama en Tacna (Pérez y Balta datos no publicados), en la presente evaluación fueron refgistrados en tyllansiales del Cerro Lechuza, en la Península de Paracas. Sin embargo, en los tyllansiales de la isla Sangayán y en la Loma de arbustos de Morro Quemado se registró a $P$. angustidigitus. El gecónido del sur $P$. gerrhopygus está presente en todos los hábitats disponibles en la zona norte de la RNP, a excepción de las Playas, pero aún no habría llegado a la zona centro y sur de esta Reserva, y por lo tanto, $P$. angustidigitus podría estar presente en lugares como la Loma de Morro Quemado.

Por otro lado, la ausencia de registros de P. angustidigitus en el hábitat de Playas seria un sesgo en nuestros datos, ya que se han reportado anteriormente individuos de esta especie en playas de la RNP como El Cangrejal (Catenazzi \& Donnelly 2007b) y La Mina (Pérez datos no publicados). Cabe destacar que la baja superposición en el uso de hábitats registrada entre $P$. angustidigitus y $P$. gerrhopygus se debe principalmente a la baja amplitud en el nicho espacial de $P$. angustidigitus, que fue registrado únicamente en dos hábitats. Por lo tanto, P. gerrhopygus presenta un mayor rango de posibles hábitats a ocupar.

Debido al reducido número de datos para el análisis del nicho temporal de ambas especies de gecónidos, se observa un sesgo considerable en los valores de amplitud y superposición. Sin embargo, se puede observar una semejanza en sus horarios de actividad que pueden ser explicados a partir de su proximidad filogenética, como se ha registrado en otras especies de Phyllodactylus (Werner et al. 1996), donde los patrones de actividad son muy semejantes entre especies del mismo género.

La dieta de ambas especies se caracteriza por el consumo principalmente de artrópodos, especialmente coleópteros. Este patrón ha sido observado también en otros gecónidos como $P$. lepidopygus (Pérez 2005) y $P$. reisii (Jordán 2006). A partir de la considerable amplitud del nicho trófico de $P$. angustidigitus y $P$. gerrhopygus se puede deducir una dieta generalista, como ha sido reportado para otras especies de saurios en el Desierto Costero peruano (Péfaur \& López-Tejeda 1983, Pérez \& Jhancke 1998, Pérez 2005, Pérez \& Balta 2007, Quispitúpac \& Pérez, 2009). Por otro lado, el carácter oportunista de la dieta de $P$. angustidigitus y $P$. gerrhopygus evidenciado en esta evaluación mediante la estimación de la oferta de recursos alimentarios en el medio, sólo puede ser comparada con la investigación de Microlophus peruvianus en Matarani por Péfaur y López-Tejeda (1983), donde se presentan datos de los recursos alimentarios, y determinan que $M$. peruvianus presentó una dieta oportunista. No se cuenta con otras evaluaciones semejantes, ya que las evaluaciones de dieta de saurios costeros de nuestro país no han evaluado la oferta del medio. Cabe destacar, que la dificultad en la estimación de la oferta de recursos alimentarios explica esta ausencia de datos. Adicionalmente al considerable esfuerzo de muestreo en campo, estimar la artropofauna de una localidad es una tarea compleja, ya que para registrar la real oferta de recursos alimentarios para los saurios se necesita una variedad de métodos de colecta de invertebrados. Como se observa en los resultados del presente estudio, en la estimación de invertebrados en el medio ambiente se registraron varios tipos de presa que no fueron consumidos por las dos especies de gecónidos, y adicionalmente, no se registraron en las trampas pit-fall algunos ítems importantes que si fueron consumidos. Por lo tanto, este método de estimación de la oferta de recursos alimentarios presenta algunas limitaciones.

El consumo de material vegetal por P. gerrhopygus puede explicarse debido a que esta especie fue registrada en localidades con Lomas, donde la considerable presencia de vegetación posibilitaría su consumo. Sin embargo, cabe destacar que otra especie de gecónido del mismo género P. lepidopygus no consume material vegetal (Pérez 2005), a pesar de estar presente en hábitat de Lomas de la Reserva Nacional de Lachay, que presenta una mayor riqueza y cobertura de vegetación. De igual manera, $P$. reissi en el Bosque Tropical de Tumbes no consumiría material vegetal (Jordán 2006), a pesar de ser una zona con una cobertura vegetal considerable.

Las larvas de insectos fueron un item importante en la dieta de $P$. angustidigitus y $P$. gerrhopygus. Sin embargo el consumo de este tipo de presa es poco frecuente para la mayoría de especies de gecónidos, ya que estos presentarían un tipo de estrategia de forrajeo de emboscada (sit-and-wait foraging), caracterizada por el consumo de presas con una alta movilidad (Huey \& Pianka 1981). La relativa reducida oferta de recursos alimentarios en el Desierto de Paracas podría explicar este resultado. Cabe mencionar que otras especies del género Phyllodactylus del Desierto Costero peruano consumen larvas de insectos, como el caso de $P$. lepidopygus en la Reserva Nacional de Lachay (Pérez 2005). Por lo tanto, $P$. angustidigitus y $P$. gerrhopygus presentarían una estrategia de forrajeo intermedia entre forrajeador activo (widely foraging) y forrajeador de emboscada, que involucraría la búsqueda activa de alguna de sus presas.

La semejanza entre las dietas de $P$. angustidigitus y $P$. gerrhopygus es evidente basados en la similitud de las variables analizadas (por ejemplo: número de tipo de ítems alimentarios, número de presas consumidas, tamaño de ítems consumidos y composición). Por otro lado, según los resultados de las simulaciones efectuadas no se observa alguna reducción en la superposición en las dietas de $P$. angustidigitus y $P$. gerrhopygus. Por lo tanto, a pesar que los coleópteros son una de las principales presas para ambas especies de gecónidos, no se produciría una competencia debido a la considerable abundancia de este orden de insectos en el medio ambiente.

Estas semejanzas en las dietas de $P$. angustidigitus y $P$. gerrhopygus pueden ser producto en primer lugar de las condiciones extremas que enfrentan en el árido desierto de Paracas, que reducirían la oferta de recursos, y en segundo lugar por la cercanía filogenética ya mencionada entre estas especies, que impone limitaciones morfo-fisiológicas que se reflejarían en las similitudes de sus dietas. 
La cercanía filogenética entre $P$. angustidigitus y $P$. gerrhopygus también produciría semejanzas en el nicho temporal (horarios de actividad), que sumadas a las condiciones de extrema aridez del Desierto de Paracas, resultan en similitudes importantes en sus nichos trófico y espacial. Estas importantes similitudes en el uso de los recursos del medio, teóricamente podrían derivar en una potencial competencia entre estos dos gecónidos. Considerando estos antecedentes, así como la amplia distribución de $P$. gerrhopygus, comparada con $P$. angustidigitus, la gran variedad de hábitat donde $P$. gerrhopygus está presente en el Desierto Costero peruano, y tomando en cuenta la hipótesis propuesta del reciente ingreso de $P$. gerrhopygus en la RNP, se evidencia un potencial riesgo para las poblaciones de $P$. angustidigitus ante una eventual competencia.

La información proporcionada en este trabajo recalca la necesidad de diseñar una adecuada estrategia de conservación para garantizar el estado de conservación de P. angustidigitus; en particular en la Reserva Nacional de Paracas, área natural protegida por el Estado y donde la única población conocida de esta especie se ubica. Esta necesidad toma mayor premura porqué la RNP está expuesta a fuertes impactos antrópicos que afectan a la comunidad de fauna silvestre, especialmente a especies poca movilidad y endémicas de esta reserva como $P$. angustidigitus.

\section{Agradecimientos}

A Emilio Fuentes, Juan Carlos Heaton y Alberto Guanilo por la ayuda en el trabajo de campo. A Eliana Quispitúpac por la ayuda en la identificación de algunos insectos. A Vanderlaine Menezes por las sugerencias a este artículo. Al personal de la Reserva Nacional de Paracas por el apoyo logístico, en especial a Luis Paz Soldán y Víctor Quispe. A Raúl Sánchez y Cecilia Rivas del Grupo de Estudios Ambientales del Perú (GEA-Perú) y a World Wildlife Fund - Oficina Programa Perú (WWF-OPP) por el financiamiento de la etapa de campo del proyecto.

\section{Literatura citada}

Aguilar C., M. Lundberg, K. Siu-Ting \& M.E. Jiménez. 2007. Nuevos registros para la herpetofauna del departamento de Lima, descripción del renacuajo de Telmatobius rimac Schmidt, 1954 (Anura: Ceratophrydae) y una clave de los anfibios. Revista Peruana de Biología 14(2): 209-216.

Catenazzi A. \& M.A. Donnelly. 2007a. Distribution of geckos in northern Peru: Long-term effect of strong ENSO events? Journal of Arid Environments 71 (2007): 327-332.

Catenazzi A. \& M.A. Donnelly. 2007b. The Ulva connection: marine algae subsidize terrestrial predators in coastal Peru. Oikos 116: $75-86$

Carrillo N., D. Rothenstein, A. Salas \& Y. Werner. 1990. Radiation and convergence among desert geckos: Phyllodactylus species resembling both Ptyodactylus and Stenodactylus. Amphibia - Reptilia 11: 1-13.

Carrillo N. \& J. Icochea. 1995. Lista taxonómica preliminar de los Reptiles vivientes del Perú. Publicaciones del Museo de Historia Natural UNMSM. Serie A: Zoología. 49: 1-27.

Dixon J. \& R. Huey. 1970. Systematic of the lizards of the gekkonidae genus Phyllodactylus of mainland South America. Contribution in Science The Natural History Museum of Los Angeles. 192: 1-78.

Donoso-Barros, R. 1966. Reptiles de Chile. Ediciones Universidad de Chile. Santiago. $458+$ cxlvi.
Dunham A. E. 1983. Realized niche overlap, resources abundance, and intensity of interspecific competition. En: Huey, R., E. Pianka \& T. Schonener. 1983. Lizard Ecology: studies of a model organism. Harvard University Press. Pp. 261-280.

Krebs C. 1999. Ecological Methodology. Addison Wesley Longman.

Gotelli N.J. and G.L. Entsminger. 2010. EcoSim: Null models software for ecology. Version 7. Acquired Intelligence Inc. y Kesey-Bear. Jericho, VT 05465. <http://garyentsminger. com/ecosim.htm> (acceso 23/11/10).

Huey R. 1979. Parapatry and niche complementarity of Peruvian desert geckos (Phyllodactylus): the ambiguous role of competition. Oecologia 38: 249-259.

Huey, R. B.\& E. R. Pianka. 1981. Ecological consequences of foraging mode. Ecology, 62: 991-999.

INRENA(Instituto Nacional de Recursos Naturales). 2002. Reserva Nacional de Paracas. Plan Maestro 2003-2007. Pisco: INRENA. RJ 4652002 INRENA. 192 p.

Jordán J. 2006. Dieta de Phyllodactyllus reissi (Sauria:Gekkonidae) en la Zona Reservada de Tumbes, Perú. Revista Peruana de Biología 13(1): 121 - 123.

Núñez H. \& F. Jaksic. 1992. Lista comentada de los reptiles terrestres de Chile continental. Bol. Mus. Nac. Hist. Nat. Chile 43: 73-91.

Péfaur J. \& E. López-Tejeda. 1983. Ecological notes on the lizard Tropidurus peruvianus in southern Peru. Journal of Arid Environments. 6:155-160.

Pérez Z., J. 2005. Ecologia de Duas Espécies de Lagartos Simpatricos em uma Formação Vegetal de Lomas no Deserto Costeiro Peruano Central. Dissertação de Mestrado. Universidade do Estado do Rio de Janeiro (UERJ). Rio de Janeiro. Brasil.

Pérez J. \& K. Balta. 2007. Ecología de la comunidad de saurios diurnos de la Reserva Nacional de Paracas, Ica, Perú. Revista Peruana de Biología. 13(3): 169-176.

Pérez Z., J. y J. Jhancke. 1998. Saurios como consumidores de ectoparásitos de aves guaneras. Boletín del Instituto del Mar de Perú. 81-86.

Pianka E. 1986. Ecology and Natural History of Desert Lizards. Princeton University Press. New Jersey.

Powell R., J. S. Parmelee, M.A. Rice \& D.D. Smith. 1990. Ecological observations on Hemidactylus brooki haitianus Meerwarth (Sauria:Gekkonidae) from Hispaniola. Caribean Journal of Science 26: 67-70.

Quispitúpac E. \& J. Pérez Z. 2009. Dieta de la lagartija de las playas Microlophus peruvianus en la playa Santo Domingo. Reserva Nacional de Paracas, Ica. Revista Peruana de Biología 15(2): 129-130.

Werner Y., N. Carrillo de Espinoza, R.B. Huey, et al. 1996. Observations on body temperatures of some neotropical desert geckos (Reptilia: Sauria: Gekkoninae). Cuadernos de Herpetología, 10 (1-2): 62-67.

Winemiller K.O. \& E.R. Pianka. 1990. Organization in natural assemblages of desert lizards and tropical fishes. Ecological Monographs 60: 27-55.

Zevallos H., E. López, L. Villegas, et al. 2002. Distribución de los reptiles de Arequipa, sur del Perú. Dilloniana 2(1): 27-34.

Zar J. H. 1999. Biostatistical analysis. 4 ed. Upper Saddle River. Prentice Hall. 
